\title{
Performance analysis of irrigation channels with distributed control*
}

\author{
Y. Li and B. De Schutter
}

If you want to cite this report, please use the following reference instead:

Y. Li and B. De Schutter, "Performance analysis of irrigation channels with distributed control," Proceedings of the 2010 IEEE International Conference on Control Applications, Yokohama, Japan, pp. 2148-2153, Sept. 2010.

Delft Center for Systems and Control Delft University of Technology

Mekelweg 2, 2628 CD Delft

The Netherlands

phone: +31-15-278.24.73 (secretary)

URL: https: //www.dcsc.tudelft.nl

*This report can also be downloaded via https://pub. deschutter.info/abs/10_032.html 


\title{
Performance Analysis of Irrigation Channels with Distributed Control
}

\author{
Yuping Li and Bart De Schutter
}

\begin{abstract}
For a string of pools with distant-downstream control, the internal time-delay for water transport from upstream to downstream not only limits the local control performance of regulating water-levels at setpoints and rejecting offtake disturbances in each pool, but also impacts the global performance of managing the water-level error propagation and attenuating the amplification of control actions in the upstream direction. A distributed control scheme which inherits the interconnection structure of the plant is studied. It is shown that the decoupling terms in the controller helps to improve global closed-loop performance by decreasing the low-frequency gain of the closed-loop coupling. Moreover, they compensate for the influence of the time-delay by imposing extra phase leadlag compensation in the mid-frequency range on the closed-loop coupling function.
\end{abstract}

\section{INTRODUCTION}

Water is becoming a scarce resource all over the world. Irrigation accounts for $70 \%$ of water usage [1]. Fig. 1 shows the topview of a typical irrigation network. Water is drawn

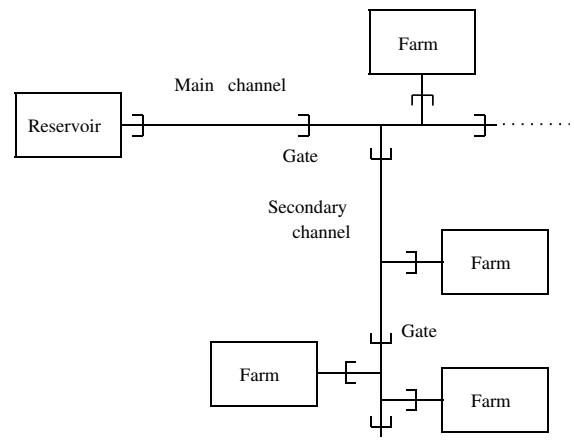

Fig. 1. Topview of an irrigation network

from the reservoir and distributed through the main channel and many secondary channels to farms. Along the channels, mechanical gates are installed to regulate the flow, as shown in Fig. 2. A stretch of water between two neighbouring gates is called a pool. An irrigation network is largely gravity-fed (i.e. there is no pumping); to satisfy water-demands from farms and to decrease water wastage, the water-levels in the pools should be regulated to certain setpoints. Since most farms sit at the downstream ends of pools, it is more important to control downstream water-levels. To avoid the excessive communication load for large-scale system, decentralised control is preferred to centralised control. In practice,

Y. Li and B. De Schutter are with the Delft Center for Systems and Control, Delft University of Technology, Mekelweg 2, 2628 CD Delft, the Netherlands. Email: \{yuping.li,b.deschutter\}etudelft.nl

The work was supported in part by the Australian Research Council (Linkage Grant LP0349134), the European 7th framework STREP project HD-MPC, the Delft Research Center NGI, and the BSIK project NGI.

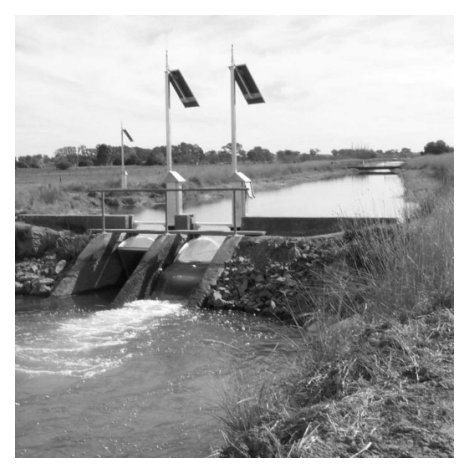

Fig. 2. An irrigation channel (Source: Rubicon Systems Australia Pty. Ltd)

a distant-downstream control structure (i.e. use upstream gate to control downstream water-level of a pool) is implemented for good management of water service and water distribution efficiency [2]. Further, an irrigation channel is a system presenting strong interactions between pools, i.e. the flow into a pool is equivalent to the flow out of the neighbouring upstream pool. When offtakes occur at downstream pool, one could see amplification of the control action (e.g. flow over upstream gates) and water-level error propagation towards upstream, see [3], [9]. Therefore, control objectives for largescale irrigation network involve: locally, setpoints regulation, rejection of offtake disturbances, avoiding excitement of dominant waves and, globally, management of the waterlevel error propagation and attenuation of the amplification of control action in the upstream direction. As shown in [9], there exists a tradeoff between the local and the global control performance. To cope with such a tradeoff, a distributed control scheme that inherits the interconnecting structure of the plant is suggested in [3], [4]. Such a distributed control scheme presents performance advantage over decentralised feedback with feedforward control [5].

In fact, one big issue in control design for an irrigation network comes from the time-delay in each pool, i.e. the time for transporting water from the upstream gate to the downstream gate. In this paper, the impact of the internal time-delays on the local and global control performance is analysed. Further, we discuss how the distributed control scheme compensates for such impact. Although the paper focuses on irrigation networks, the discussion can be extended to many practical networks that involve internal timedelay. The paper is organised as follows. Section II briefly introduces modelling of an irrigation channel and designing of the distributed controller. In Section III, discussions are made on how the distributed control scheme manages the water-level error propagation and attenuates the amplification 
of control actions in the upstream direction. Section IV summarises the paper.

\section{MODELLING OF A CHANNEL AND DESIGNING OF DISTRIBUTED CONTROLLER}

Fig. 3 shows an irrigation channel with a special structured distributed control, i.e. the information flow is unidirectional: from controller $K_{i+1}$ to controller $K_{i}$. When water offtakes occur in a pool, such an interconnection structure confines the water-level error propagation and amplification of control action in the upstream pools. Hence, such a control scheme avoids the requirement of water storage at the downstream end of the channel.

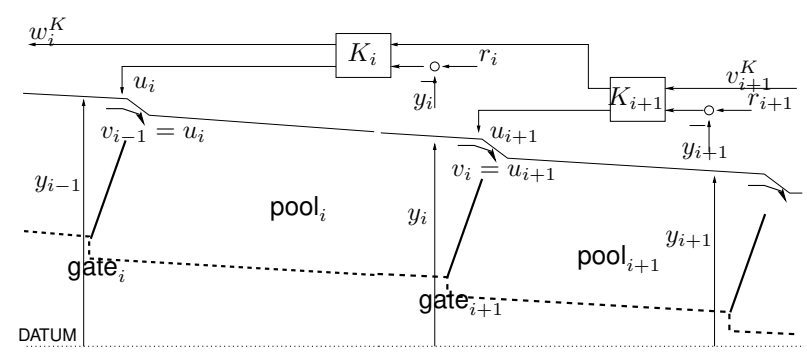

Fig. 3. Distributed control of an open water channel

\section{A. Plant model}

A simple model of the water-level in pool $_{i}$ can be obtained by conservation of mass [3], [6]:

$$
\alpha_{i} \dot{y}_{i}(t)=u_{i}\left(t-\tau_{i}\right)-v_{i}(t)-d_{i}(t),
$$

where $u_{i}$ is the flow over the upstream gate, $v_{i}$ the flow over the downstream gate, $d_{i}$ models the offtake load-disturbances from pool $_{i} ; \tau_{i}$ is the transport delay of water from upstream gate to downstream gate of the pool, and $\alpha_{i}$ a measure of the pool surface area. Note the interconnection $v_{i}=u_{i+1}$, i.e. the flow out from pool ${ }_{i}$ equals the flow into pool ${ }_{i+1}$. Taking Laplace transform, yields

$$
P_{i}: y_{i}(s)=\frac{1}{s \alpha_{i}}\left(e^{-s \tau_{i}} u_{i}-v_{i}-d_{i}\right)(s) .
$$

\section{B. Designing of the distributed controller}

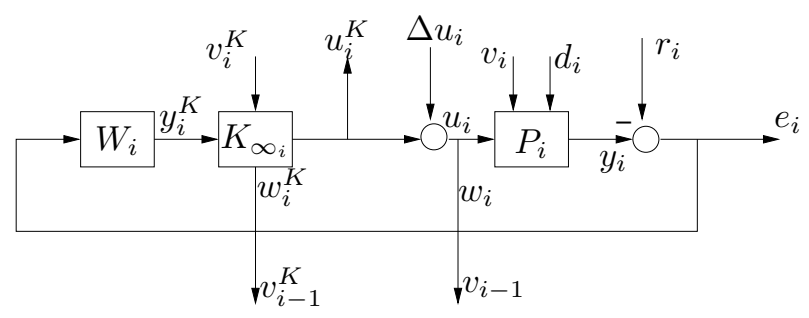

Fig. 4. Localised portion of distributed controller design

Fig. 4 shows a localised portion of a channel under distributed distant-downstream control, where $P_{i}$ is the nominal model (1) for $\operatorname{pool}_{i}$, and $K_{i}$ in Fig. 3 is split into a loop-shaping weight $W_{i}$ and a compensator $K_{\infty_{i}}$ (with $y_{i}^{K}$ and $u_{i}^{K}$, input from and output to the shaped plant, respectively). Note the constraint on the interconnection between controllers $v_{i}^{K}=w_{i+1}^{K}$. Designing of the distributed controller consists of the following three steps, which are consistent with the well-known $\mathcal{H}_{\infty}$ loop-shaping approach [11].

1) Design $W_{i}$ to shape $P_{i}$ based on local performance. Typical offtakes $d_{i}$ are step disturbances; based on the internal model principle [7], a simple selection could be $W_{i}=\frac{\kappa_{i}}{s}$ for zero steady-state water-level error. For robust stability, $\kappa_{i}$ is selected such that the local crossover frequency $\omega_{c_{i}} \leq 1 / \tau_{i}$ (see [8]). Denote $z_{i}:=\left(e_{i}, u_{i}^{K}\right)^{T}$ and $n_{i}:=\left(r_{i}, \Delta u_{i}, d_{i}\right)^{T}$, with $r_{i}$ the water-level setpoint and $\Delta u_{i}$ modelling additional uncertainty in flow over gate ${ }_{i}$. For a channel of $N$ pools, Let $G_{s}:=\left(G_{s_{1}}, \ldots, G_{s_{N}}\right)$ denote the interconnection of the shaped plant

$$
\begin{aligned}
& G_{s_{i}}:=\left(\begin{array}{c}
v_{i} \\
n_{i} \\
u_{i}^{K}
\end{array}\right) \mapsto\left(\begin{array}{c}
w_{i} \\
z_{i} \\
y_{i}^{K}
\end{array}\right) \\
& =\left[\begin{array}{ccc}
0 & \left.\begin{array}{ccc}
0 & 1 & 0
\end{array}\right) \\
\left(\begin{array}{ccc}
\frac{1}{s \alpha_{i}} \\
0
\end{array}\right)\left(\begin{array}{ccc}
1 & \frac{e^{-s \tau_{i}}}{-s \alpha_{i}} & \frac{1}{s \alpha_{i}} \\
0 & 0 & 0
\end{array}\right) & \left.\begin{array}{c}
\frac{e^{-s \tau_{i}}}{-s \alpha_{i}} \\
1
\end{array}\right) \\
\frac{W_{i}}{s \alpha_{i}} & \left(W_{i} \frac{e^{-s \tau_{i}} W_{i}}{-s \alpha_{i}} \frac{W_{i}}{s \alpha_{i}}\right) & \frac{e^{-s \tau_{i} W_{i}}}{-s \alpha_{i}}
\end{array}\right]
\end{aligned}
$$

with $v_{i}=w_{i+1}$ and boundary condition $v_{N}=0$. Note that such a boundary condition is possible with distantdownstream control.

2) Synthesise $K_{\infty_{i}}$ to cope with the tradeoff between local performance and closed-loop coupling. ${ }^{1}$ Let $K_{\infty}:=\left(K_{\infty_{1}}, \ldots, K_{\infty_{N}}\right)$ denote the interconnection of

$$
K_{\infty_{i}}:=\left(\begin{array}{c}
v_{i}^{K} \\
y_{i}^{K}
\end{array}\right) \mapsto\left(\begin{array}{c}
w_{i}^{K} \\
u_{i}^{K}
\end{array}\right)
$$

with $v_{i}^{K}=w_{i+1}^{K}$ and boundary condition $v_{N}^{K}=0$; and let $H\left(G_{s}, K_{\infty}\right)$ denote the closed-loop transfer function from $\left(n_{1}, \ldots, n_{N}\right)^{T}$ to $\left(z_{1}, \ldots, z_{N}\right)^{T}$. The synthesis problem is formulated as

$$
\begin{gathered}
\min _{\substack{K_{\infty} \in \mathcal{K}_{\text {syn }} \\
\text { subject to }}} \\
\left\|H\left(G_{s}, K_{\infty}\right)\right\|_{\infty}<\gamma
\end{gathered}
$$

where $\mathcal{K}_{\text {syn }}$ represents the set of stablising $K_{\infty}$ 's. Note that we use $\|\cdot\|_{\infty}$ to denote the $\mathcal{H}_{\infty}$ norm of a transfer function. Such a structured optimisation problem can be solved by employing the technique in [10], see [4].

3) The final distributed controller is then given by

$$
K_{i}:=\left(\begin{array}{c}
v_{i}^{K} \\
e_{i}
\end{array}\right) \mapsto\left(\begin{array}{c}
w_{i}^{K} \\
u_{i}
\end{array}\right)=K_{\infty_{i}}\left[\begin{array}{cc}
1 & 0 \\
0 & W_{i}
\end{array}\right] .
$$

\section{CLOSED-LOOP PERFORMANCE}

For distant-downstream control, the internal time-delay $\tau_{i}$ limits the local performance. For example, the local bandwidth limit of $1 / \tau_{i}$ is previously considered in the selection

\footnotetext{
${ }^{1}$ For local performance, one considers $e_{i}$ to be small; while closed-loop coupling is cause by control action $u_{i}$ to compensate $e_{i}$. As shown in [3], [9], for purely decentralised feedback control, $T_{r_{i} \mapsto e_{i}}+T_{d_{i} \mapsto u_{i}} e^{-s \tau_{i}}=$
} 1 . 
of the weight gain, $\kappa_{i}$. In this section, the influences of $\tau_{i}$ on the closed-loop coupling are discussed. It is shown that such time-delays, not only make it difficult to manage the waterlevel error propagation, but also cause the amplification of control action, in the upstream direction. Further, analysis is made on how the distributed control compensates for such influences.

\section{A. The impact of $\tau_{i}$ on global closed-loop performance}

From (1), for a channel of $N$ pools

$$
\begin{aligned}
& \left(\begin{array}{c}
y_{1} \\
\vdots \\
y_{N-1} \\
y_{N}
\end{array}\right)=\left[\begin{array}{cccc}
G_{1} & \tilde{G}_{1} & & \\
& \ddots & \ddots & \\
& & G_{N-1} & \tilde{G}_{N-1} \\
& & & G_{N}
\end{array}\right]\left(\begin{array}{c}
u_{1} \\
\vdots \\
u_{N-1} \\
u_{N}
\end{array}\right) \\
& +\left[\begin{array}{ccc}
\tilde{G}_{1} & & \\
& \ddots & \\
& & \tilde{G}_{N}
\end{array}\right]\left(\begin{array}{c}
d_{1} \\
\vdots \\
d_{N}
\end{array}\right)
\end{aligned}
$$

where $G_{i}=\frac{1}{s \alpha_{i}} e^{-s \tau_{i}}$ and $\tilde{G}_{i}=-\frac{1}{s \alpha_{i}}$. As previously mentioned, it is reasonable to assume $v_{N}=0$ as boundary condition for synthesis of the distributed controller under distant-downstream control. The distributed controller is represented by

$$
\begin{array}{rll}
K_{1}: & u_{1}=\left[\begin{array}{ll}
K_{1}^{21} & K_{1}^{22}
\end{array}\right]\left(\begin{array}{c}
w_{2}^{K} \\
e_{1}^{K}
\end{array}\right) \\
K_{i}: & \left(\begin{array}{c}
w_{i}^{K} \\
u_{i}
\end{array}\right)=\left[\begin{array}{ll}
K_{i}^{11} & K_{i}^{12} \\
K_{i}^{21} & K_{i}^{22}
\end{array}\right]\left(\begin{array}{c}
w_{i+1}^{K} \\
e_{i}
\end{array}\right) \\
& \text { for } i=2, \ldots, N-1 \\
K_{N}: & \left(\begin{array}{c}
w_{N}^{K} \\
u_{N}
\end{array}\right)=\left[\begin{array}{c}
K_{N}^{12} \\
K_{N}^{22}
\end{array}\right] e_{N}
\end{array}
$$

This gives the general form of the distributed controller $K$ :

$$
\left(\begin{array}{c}
u_{1} \\
\vdots \\
u_{N}
\end{array}\right)=\left[\begin{array}{ccc}
K_{11} & \cdots & K_{1 N} \\
& \ddots & \vdots \\
& & K_{N N}
\end{array}\right]\left(\begin{array}{c}
e_{1} \\
\vdots \\
e_{N}
\end{array}\right)
$$

where for $i=1, \ldots, N, K_{i i}=K_{i}^{22}$, which takes care of local performance, and the additional decoupling terms

$$
\begin{aligned}
K_{i, i+1} & =K_{i}^{21} K_{i+1}^{12}, \\
K_{i j} & =K_{i}^{21}\left(\prod_{k=i+1}^{j-1} K_{k}^{11}\right) K_{j}^{12} \text { for } j>i+1 .
\end{aligned}
$$

Note that $e_{i}=r_{i}-y_{i}$. Then the closed-loop relationship between water-level errors and offtake disturbances is:

$$
\left(\begin{array}{c}
e_{1} \\
\vdots \\
e_{N}
\end{array}\right)=\left[\begin{array}{ccc}
M_{11} & \cdots & M_{1 N} \\
& \ddots & \vdots \\
& & M_{N N}
\end{array}\right]\left(\begin{array}{c}
d_{1} \\
\vdots \\
d_{N}
\end{array}\right)
$$

where for $i=1, \ldots, N, M_{i i}=-\tilde{G}_{i}\left(1+G_{i} K_{i i}\right)^{-1}$ and for $j \geq i+1$

$$
M_{i j}=M_{i i} \sum_{k=i+1}^{j}\left(K_{i+1, k}-K_{i k} e^{-s \tau_{i}}\right) M_{k j} .
$$

We see that the closed-loop transfer matrix is uppertriangular, hence the multivariable system inherits the local stabilities. That is, the multivariable system is stable if and only if all monovariable systems are stable. Since all the lower off-diagonal entries are null, even for model mismatch, robustness is also inherited from local systems. A perfect decoupling is achieved if for all $j>i$,

$$
K_{i+1, j}-K_{i j} e^{-s \tau_{i}}=0 .
$$

This requires $K_{i j}=K_{i+1, j} e^{s \tau_{i}}$, which is non-causal and hence impractical.

Next, analysis of global closed-loop performance is made on the two typical coupling properties of a (distantdownstream) controlled irrigation channel: water-level error propagation and amplification of control action. Assume only $d_{N}$ occurs in the system, while $d_{i}=0$ for $i=1, \ldots, N-1$. Then from (6),

$$
\begin{aligned}
& T_{e_{i+1} \mapsto e_{i}}:=M_{i, N} M_{i+1, N}^{-1} \\
& =M_{i i}\left(K_{i+1, i+1}-e^{-s \tau_{i}} K_{i, i+1}\right)+ \\
& M_{i i} \sum_{k=i+2}^{N}\left(K_{i+1, k}-K_{i k} e^{-s \tau_{i}}\right) M_{k N} \\
& \left(M_{i+1, i+1} \sum_{k=i+2}^{N}\left(K_{i+2, k}-K_{i+1, k} e^{-s \tau_{i+1}}\right) M_{k N}\right)^{-1} .
\end{aligned}
$$

Small $\left\|T_{e_{i+1} \mapsto e_{i}}\right\|_{\infty}$ (e.g. $\left.\ll 1\right)$ represents a good management of the water-level error propagation.

Remark 1: For the case of a string of identical pools with purely decentralised feedback control (i.e. $K=\operatorname{diag}\left(K_{i i}\right)$ ), $T_{e_{i+1} \mapsto e_{i}}=M_{i i} K_{i+1, i+1}$. If the selected $K_{i i}$ 's are identical for all $i=1, \ldots, N$, then $\left\|T_{e_{i+1} \mapsto e_{i}}\right\|_{\infty}>1$ (see [3], [9]). Such a strategy, i.e. designing $K_{i i}$ only based on local control performance, creates very strong coupling between loops (since $\left\|T_{e_{i+1} \mapsto e_{i}}\right\|_{\infty}$ occurs at the same frequency for all $i)$. Instead, to decouple the interaction between pools, one can design $K_{i i}$ 's such that the downstream closed-loop be slower than the upstream ones. ${ }^{2}$ However, it is nontrivial to cope with the tradeoff between local performance and closedloop decoupling by simply tuning the feedback controller. In contrast, the resulted distributed controller, by taking the three steps in Section II, optimises a measure of the global performance, accounting for such a tradeoff.

From (4) and (6), the coupling of control actions responding to $d_{N}$ is

$$
T_{u_{i+1} \mapsto u_{i}}:=\sum_{k=i}^{N} K_{i k} M_{k N}\left(\sum_{k=i+1}^{N} K_{i+1, k} M_{k N}\right)^{-1} .
$$

The following discussion shows that $\left\|T_{u_{i+1} \mapsto u_{i}}\right\|_{\infty}>1$.

For an irrigation channel with purely decentralised feedback control, i.e. $K$ in (4) being diagonal, $T_{u_{i+1} \mapsto u_{i}}=$ $M_{i i} K_{i i}=-\tilde{G}_{i} K_{i i}\left(1-\tilde{G}_{i} K_{i i} e^{-\tau_{i} s}\right)^{-1}$. Note that $\tilde{G}_{i} K_{i i}$ involves two integrators. ${ }^{3}$ Applying Lemma 9.3 of [7], it is straightforward to prove $\left\|T_{u_{i+1} \mapsto u_{i}}\right\|_{\infty}>1$.

Generally, under distant-downstream control (i.e. without the constraints that $K$ in (4) be diagonal), to compensate

\footnotetext{
${ }^{2}$ Such a scheme is similar as the one suggested in [12] for the control of a platoon of vehicles, that string instability can be avoided at the expense of successively more aggressive control laws with linearly increasing gains.

${ }^{3}$ As previously discussed, for zero steady-state water-level error, an integrator is involved in $K_{i i}$.
} 


\begin{tabular}{c|c|c|c}
\hline \hline$i$ & $\tau_{i}$ & $\alpha_{i}$ & $\psi_{i}$ \\
\hline 1 & $6 \mathrm{~min}$ & $10344 \mathrm{~m}^{2}$ & $0.349 \mathrm{rad} / \mathrm{min}$ \\
2 & $25 \mathrm{~min}$ & $39352 \mathrm{~m}^{2}$ & $0.084 \mathrm{rad} / \mathrm{min}$ \\
3 & $15 \mathrm{~min}$ & $26317 \mathrm{~m}^{2}$ & $0.140 \mathrm{rad} / \mathrm{min}$ \\
\hline
\end{tabular}

TABLE I

POOL MODEL PARAMETERS: DELAY $\left(\tau_{i}\right)$, SURFACE AREA $\left(\alpha_{i}\right)$ AND WAVE FREQUENCY $\left(\psi_{i}\right)$

the influence of the internal time-delay, the amplification of control action in the upstream direction is unavoidable. This is shown in Fig. 5. Initially, the system is at steady-state.

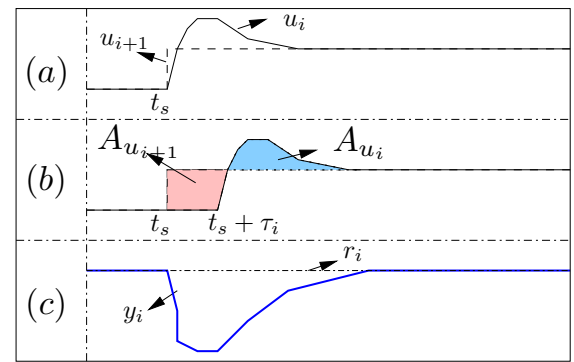

Fig. 5. Control actions for zero steady-state water-level error

At time $t_{s}$, the flow out of pool $_{i}$ increases, see the change of $u_{i+1}$ (the dashed line in Fig. 5(a)). To compensate for the influence of $u_{i+1}$ on $y_{i}$, the flow into the pool, $u_{i}$, also increases (the solid line in Fig. 5(a)). However, the influence of $u_{i}$ on the downstream water-level $y_{i}$ will be $\tau_{i}$ (min) later than that of $u_{i+1}$ on $y_{i}$ (see Fig. 5(b)). For zero steady-state error of $y_{i}$ from $r_{i}$ (see Fig. 5(c)), from (1), $u_{i}$ should be greater than $u_{i+1}$ for some time such that the area of $A_{u_{i}}$ is equivalent to the area of $A_{u_{i+1}}$. Hence, $\left\|T_{u_{i+1} \mapsto u_{i}}\right\|_{\infty}>1$.

In Section III-B, the analysis focuses on the impact of the decoupling terms in the distributed controller on the closedloop performance.

\section{B. The influence of $K_{i j}(j>i)$ on closed-loop decoupling}

As discussed in Section II-B, the synthesis of $K_{\infty}$ copes with the tradeoff between the local performance and the decoupling of the closed-loop system. To see how the distributed controller compensates for the influence of internal time-delays, we study the time and frequency responses of a string of three pools with distributed control.

The three pools are taken from Eastern Goulburn No 12, Victoria, Australia. Table I gives the identified model parameters [13]. To shape the plant, we choose $W_{1}=\frac{87.206}{\mathrm{~s}}$, $W_{2}=\frac{20.8865}{s}, W_{3}=\frac{32.6255}{s}{ }^{4} \mathrm{~A} \gamma=3$ is achieved by solving the structured optimisation problem (2). The final controller is shown in Fig. 6. All the terms involve an integrator, which comes from the shaping weight. Note that $K_{12}$ has similar phase property as $K_{22}$, i.e. they both involve phase-lead-lag-lag-lead compensation around the same midfrequency range; while $K_{13}, K_{23}$ have similar phase property as $K_{33}$.

\footnotetext{
${ }^{4}$ As formerly discussed, the weight gains are chosen to set the loop-gain
} bandwidth just below $1 / \tau_{i} \mathrm{rad} / \mathrm{min}$

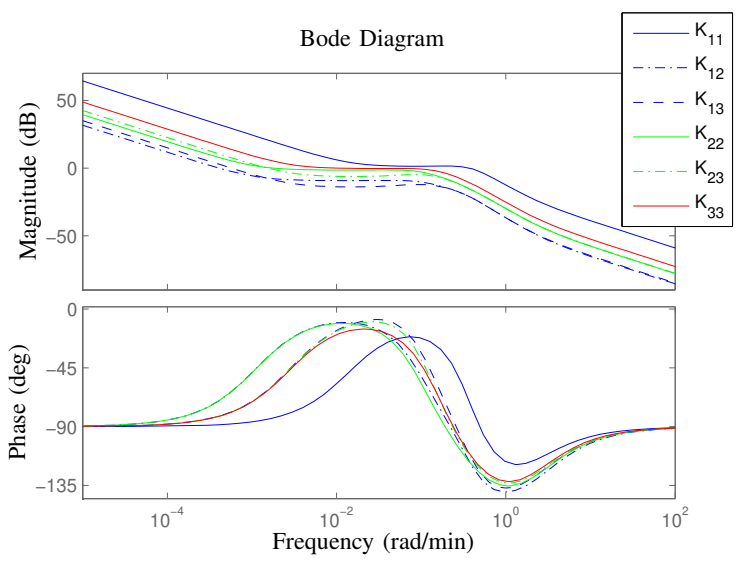

Fig. 6. The distributed controller

Fig. 7 shows the open loop-gain for pool $_{1,2,3}$. High gain at low frequency is obtained, with the bandwidths 0.0408 $\mathrm{rad} / \mathrm{min}, 0.0085 \mathrm{rad} / \mathrm{min}$ and $0.0132 \mathrm{rad} / \mathrm{min}$ respectively. Around the wave frequencies, the loop-gains are around $-20 \mathrm{~dB},-20 \mathrm{~dB}$ and $-25 \mathrm{~dB}$ respectively. This ensures no excitement of dominant waves in all the three pools.

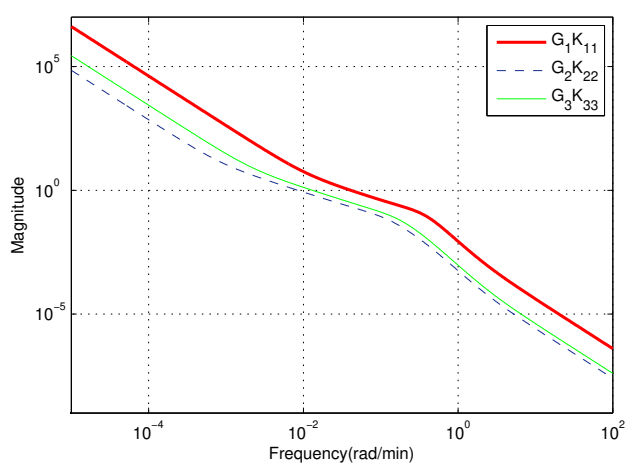

Fig. 7. Local loop-gain with the distributed controller

From (5), $K_{12}$ and $K_{23}$ have a similar structure, while $K_{13}$ involves $K_{2}^{11}$ for decoupling. The following analysis is made by checking the impact of $K_{23}{ }^{5}$ and $K_{13}$ on decoupling of the closed-loop system.

1) Impact of $K_{23}$ : The gains of $T_{d_{3} \mapsto e_{2}}$ and $T_{d_{3} \mapsto u_{2}}$, with and without $K_{23}$, are given in Fig. 8. With $K_{23}$, a lower gain in the mid-frequency range is achieved.

Fig. 9 shows that $K_{23}$ helps in decreasing $\left|T_{e_{3} \mapsto e_{2}}\right|$ and $\left|T_{u_{3} \mapsto u_{2}}\right|$ at the low and middle-frequency range, where $d_{3}$ is significant. One can thus expect a good management of the water-level error propagation and attenuation of the amplification of control action with $K_{23}$.

The time response of the close-loop system is shown in Fig. 10 and 11. In the simulation, the water-level setpoints are set as $r_{i}=10 \mathrm{~m}$, for $i=1,2,3$. Note that $\tau_{2}$ is much bigger than $\tau_{3}$; such a combination, i.e. a long upstream pool with a

\footnotetext{
${ }^{5}$ Similar impact of $K_{12}$ as that of $K_{23}$ on the closed-loop decoupling can be expected and hence the analysis is omitted here.
} 

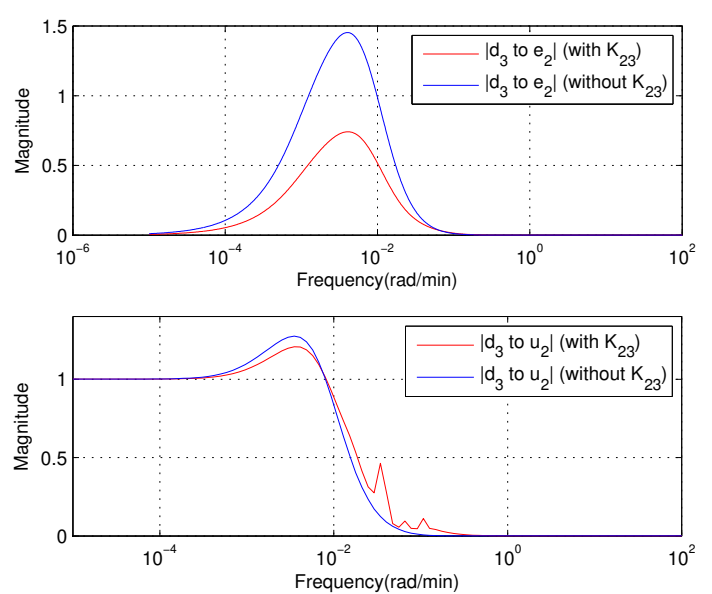

Fig. 8. $\left|T_{d_{3} \mapsto e_{2}}\right|$ (top) and $\left|T_{d_{3} \mapsto u_{2}}\right|$ (bottom), with and without $K_{23}$
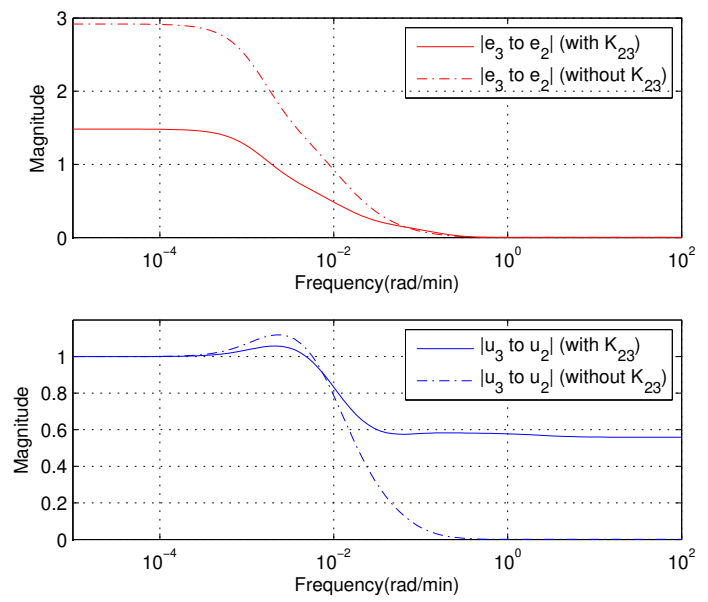

Fig. 9. Closed-loop coupling: $\left|T_{e_{3} \mapsto e_{2}}\right|$ and $\left|T_{u_{3} \mapsto u_{2}}\right|$

short downstream pool, is difficult for managing the tradeoff between the local water-level error and the amplification of control action. ${ }^{6}$ When an offtake of $98.6 \mathrm{Ml} /$ day starts in

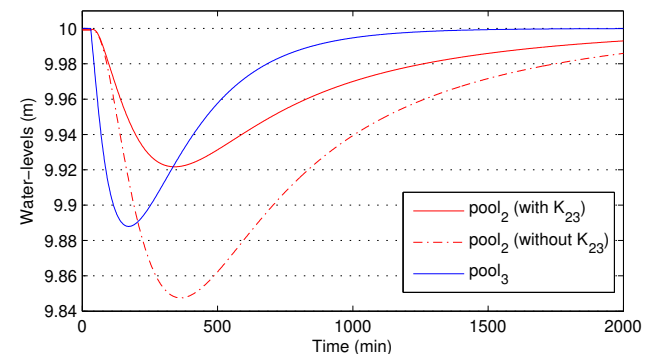

Fig. 10. Water-level error propagation: with and without $K_{23}$

pool $_{3}$ at $30 \mathrm{~min}$ till the end of the simulation scenario, the water-level error in pool 2 is better managed with $K_{23}$ operating in the system than without $K_{23}$. Indeed, with $K_{23}$, $\max _{t}\left|e_{2}(t)\right|$ decreases about $0.08 \mathrm{~m}$ (compare the red solid

${ }^{6}$ As previously discussed, to decouple the closed-loop system, one should try to make the downstream loop slower than the upstream loop. line with the red dashed line). This is important since, as discussed in Section I, in gravity-fed irrigation networks, water-levels represent the capacity to serve water-demands at the offtake points. Fig. 11 shows the upstream control actions in pool $_{2,3}$ to compensate the influence of $d_{3}$ on $e_{2}$ and $e_{3} .{ }^{7}$ With $K_{23}, u_{2}$ responds to the change of $u_{3}$ faster than without $K_{23}$ operating on the closed-loop. Note $\max _{t}\left|u_{2}(t)\right|$ is smaller with $K_{23}$, i.e. a better attenuation of the amplification of control action is obtained.

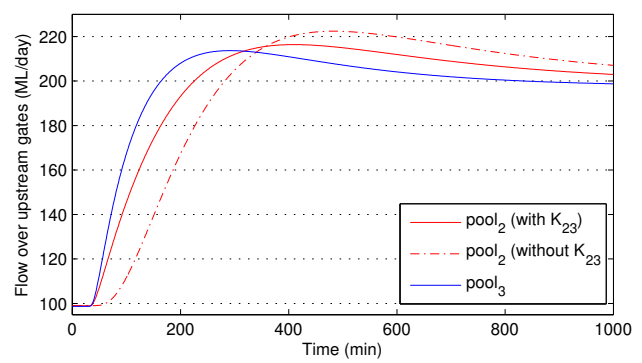

Fig. 11. Amplification of control actions: with and without $K_{23}$

2) Impact of $K_{13}$ : Fig. 12 shows $\left|T_{d_{3} \mapsto e_{1}}\right|$ and $\left|T_{d_{3} \mapsto u_{1}}\right|$, with and without $K_{13} .{ }^{8}$ With $K_{13}$, a lower gain in the low and mid-frequency range is achieved, hence better decoupling of the closed-loop system can be expected. This is confirmed by the time responses shown in Fig. 13 and 14 . When $d_{3}$ starts at $30 \mathrm{~min}$, the water-level error in pool $_{1}$
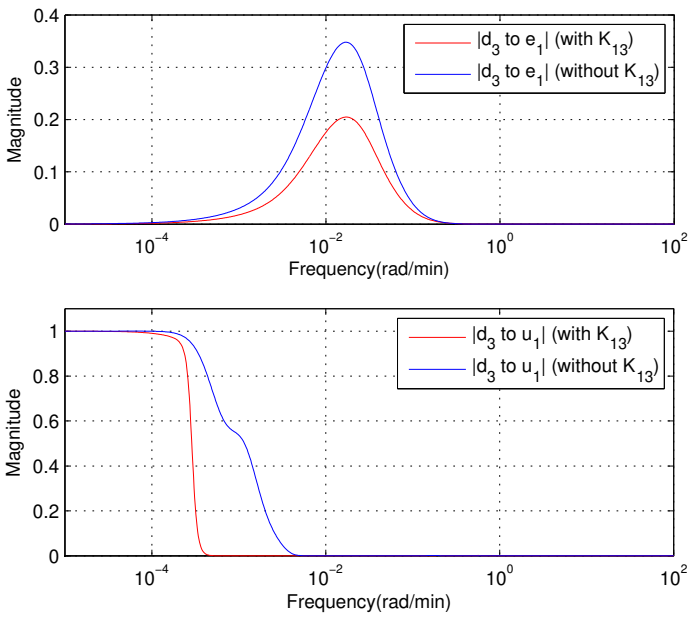

Fig. 12. $\left|T_{d_{3} \mapsto e_{1}}\right|$ (top) and $\left|T_{d_{3} \mapsto u_{1}}\right|$ (bottom), with and without $K_{13}$

is smaller with $K_{13}$ (see the green solid line in Fig. 13) operating in the system than without $K_{13}$ (the green dash-dot line). Fig. 14 shows the change of control actions in pool $_{1,2,3}$ in response to $d_{3}$. We see that with $K_{13}, u_{1}$ reacts faster to the change in $u_{2}$ than the case without $K_{13}$. Moreover, $\left\|u_{1}\right\|_{\infty}$ is smaller with $K_{13}$.

${ }^{7}$ For clarity, we zoomed in to the first 1000 mins to show the changes of the control actions when $d_{3}$ starts. Note we did the similar in Fig. 14 .

${ }^{8}$ For the case of $K_{13}=0$, it is assumed that $K_{2}^{11}=0$, while $K_{12}$ and $K_{23}$ still operate on the closed-loop. 


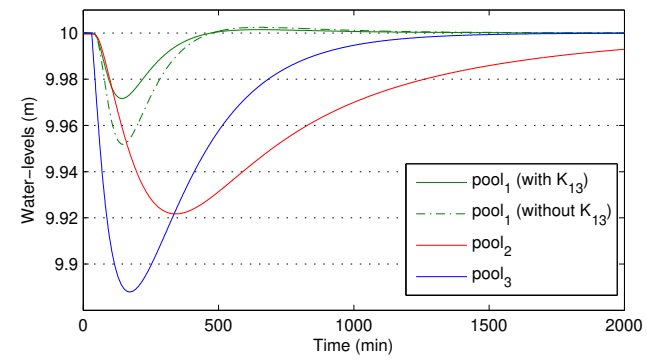

Fig. 13. Water-level error propagation: with and without $K_{13}$

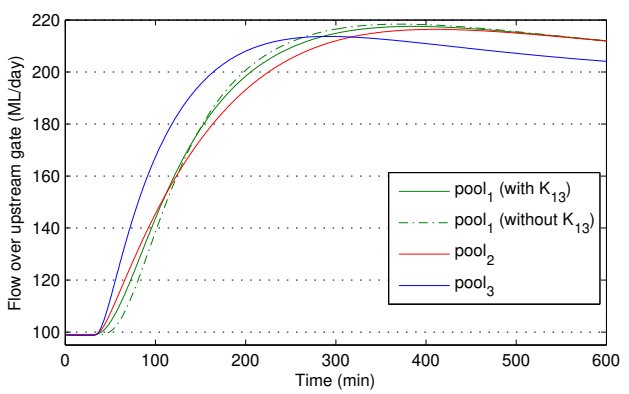

Fig. 14. Control actions: with and without $K_{13}$

3) Some remarks: The closed-loop coupling term $M_{i j}$ (see (7)) is composed of $M_{i j}^{k}:=$ $M_{i i}\left(K_{i+1, k}-K_{i k} e^{-s \tau_{i}}\right) M_{k j}$ for $k=i+1, \ldots, j$. Fig. 15 shows the impact of $K_{i k}$ on $M_{i j}^{k}$ in the above three-pool example. It is observed that

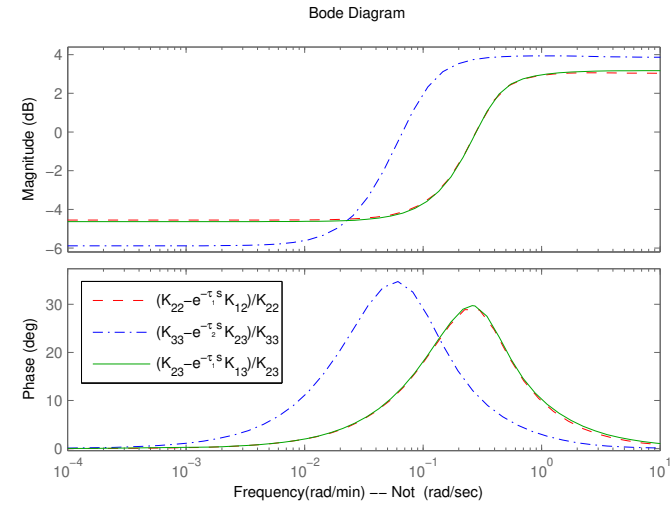

Fig. 15. The decoupling function of $K_{i k}$ for $k=i+1, \ldots, j$

1) $K_{i k}$ decreases the gain of $M_{i j}^{k}$ at low frequencies where typical offtake disturbances are significant;

2) $K_{i k}$ operates on $M_{i j}^{k}$ by imposing on $M_{i i} K_{i+1, k} M_{k j}$ an additional phase lead-lag compensation around the frequency of $1 / \tau_{i}$.

The first observation explains why with $K_{i j}$ operating on the closed-loop, a better management of water-level error propagation is achieved (see Fig. 10 and 13). Although it is difficult to directly make conclusions of global performance from the second observation, time-responses of control actions (see
Fig. 11 and 14) show that with the $K_{i j}$ 's the closed-loop predicts the influence of the internal time-delays and that the control action in response to offtake disturbance is faster than that without the $K_{i j}$ 's.

\section{SUMMARY}

An irrigation channel is a system presenting strong interactions between pools. This paper considers distantdownstream control of irrigation channels. It is shown that the internal time-delay for transportation of water from upstream to downstream of each pool not only limits the local performance, but also impacts the coupling between pools, i.e. the water-level error propagation and the amplification of control actions in the upstream direction. More specifically, we have discussed a distributed control that inherits the interaction structure of the plant. The controller is designed in a structured $\mathcal{H}_{\infty}$ loopshaping approach. The involved optimisation problem manages the tradeoff between local and global performance. Analysis shows that the distributed controller compensates the time-delay influence by decreasing the low-frequency gain of the close-loop coupling term and imposing extra phase lead-lag compensation in the midfrequency range on the closed-loop coupling term.

Based on the above observations of the function of the decoupling terms of the distributed controller, it is of interest in future research to investigate the involvement of similar components, e.g. phase lead-lag in decentralised feedforward compensators, in addition to the purely decentralised feedback controller, for a better global closed-loop performance.

\section{REFERENCES}

[1] UNESCO water report (2003). The United Nations World Water Development Report, http://www.unesco.org/water/wwap.

[2] X. Litrico and V. Fromion, "Advanced control politics and optimal performance for an irrigation canal", in Proceedings of the 2003 ECC, Cambridge, UK, 2003.

[3] M. Cantoni, E. Weyer, Y. Li, S.K. Ooi, I. Mareels and M. Ryan, "Control of large-scale irrigation networks", J. Bailliuel and P. Antsaklis (eds.), Special Issue on the Technology of Networked Control Systems, Proceedings of the IEEE, vol. 95(1), 2007, pp. 75-91.

[4] Y. Li and M. Cantoni, "Distributed controller design for open water channels", in Proceedings of the 17th IFAC World Congress, Korea, July 2008, pp. 10033-10038.

[5] E. Weyer, "Control of irrigation channels", IEEE Transactions on Control Systems Technology, vol 16(4), July 2008, pp. 664-675.

[6] E. Weyer, "System identification of an open water channel", Control Engineering Practice (IFAC), vol. 9(12), 2001, pp. 1289-1299.

[7] G. C. Goodwin, S.F. Graebe and M.E. Salgado, Control System Design, Prentice Hall, Englewood Cliffs, NJ; 2001.

[8] S. Skogestad and I. Postlethwaite, Multivariable Feedback Control, John Wiley and Sons, Chichester, UK; 1996.

[9] Y. Li, M. Cantoni and E. Weyer, "On water-level error propagation in controlled irrigation channels", In Proceedings of the 44th IEEE $C D C$, Seville, Spain, December 2005, pp. 2101-2106.

[10] C. Langbort, R. Chandra and R. D'Andrea, "Distributed control design for systems interconnected over an arbitrary graph", IEEE Transactions on Automatic Control, vol. 49(9), pp. 1502-1519.

[11] D. C. McFarlane and K. Glover, "Robust controller design using normalized coprime factor plant descriptions", Lecture Notes in Control and Information Sciences, Springer-Verlag, 1990.

[12] M. E. Khatir and E. J. Davidson, "Bounded stability and eventual string stability of a large platoon of vehicles using non-identical controllers", in Proceedings of IEEE CDC, 2004, pp. 1111-1116.

[13] S. K. Ooi, M. Krutzen and E. Weyer, "On physical and data driven modeling of irrigation channels", Control Engineering Practice (IFAC), vol. 13(4), 2001, pp. 461-471. 